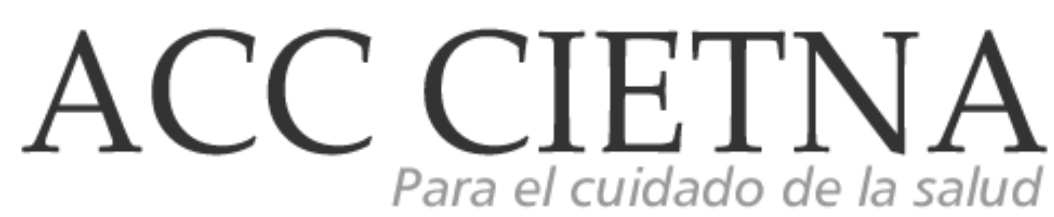

https://doi.org/10.35383/cietna.v1i1.165

\title{
La importancia de la coordinación en la Gestión del Cuidado
}

\author{
Reynaldos Katiuska ${ }^{1}$, Amaya Pavez ${ }^{2}$
}

\section{INFORMACIÓN DEL ARTÍCULO RESUMEN}

\section{Historia del artículo:}

Recibido el 12 de abril de 2013

Aceptado el 5 de octubre de 2013

\section{Palabras claves:}

Cuidado

Gestión

Enfermería
El presente escrito nos lleva a reflexionar sobre la importancia de la coordinación en la gestión del cuidado en la práctica de enfermería, una exigencia que está implícita en la declaración hecha por el Colegio de Enfermeras de Chile (2007) cuando resalta la complejidad de la atención de enfermería en "el nuevo escenario profesional, que se está generando tras este nuevo modelo de atención, requiere enfermeras capacitadas y empoderadas de nuestro rol profesional", este cambio implica aplicar diversas acciones como son la estandarización de procesos, la optimización de la comunicación e información a través de la coordinación e interacción.

\section{The importance of coordination in the Management of Care}

\section{ABSTRACT}

\section{Keywords:}

Care

Management

Nursing
This paper leads us to reflect on the importance of coordination in nursing care management, a requirement that is implicit in the statement made by the College of Nurses of Chile (2007) when it highlights the complexity of care Nursing in "the new professional scenario, which is being generated following this new model of care, requires trained and empowered nurses of our professional role", this change implies applying various actions such as the standardization of processes, the optimization of communication and information through coordination and interaction.

\footnotetext{
${ }^{1}$ Enfermera-Matrona, Ingeniero Comercial, MBA, Dra. @ Gestión Internacional de Empresas. Profesora Escuela de Enfermería de la Pontificia Universidad Católica de Chile, Santiago De Chile, Chile. Email: kreinaldlos@gmail.com

2 Enfermera-Matrona, Antropóloga Social, Magíster y Dra. en Antropología. Profesora Escuela de Enfermería de la Universidad de Santiago, Santiago De Chile, Chile. Email: pamaya@hotmail.com
} 


\section{Introducción}

En los sistemas de salud el mayor reto es la superación de la fragmentación de la organización y de la prestación de los servicios, es por ello que la coordinación del quehacer de enfermería es uno de los pilares elementales de la gestión del cuidado clínico. Sin embargo, su implementación se transforma en un desafío al tratar de conciliar la efectividad empresarial con la esencia del cuidado de la disciplina de Enfermería, de forma que la coordinación deba ser efectiva en lo administrativo $y$ en lo humano.

\section{Desarrollo}

En la dinámica de la sociedad contemporánea ha emergido la complejidad de los sistemas de salud que ha exigido afrontarlos desde una doble perspectiva: la primera acorde con su sentido de ser, la atención a las personas para favorecer su salud y la segunda de acuerdo con la lógica de las sociedades de mercado, las organizaciones de salud como empresa. De la conciliación de estas dos miradas emergen dos objetivos y a veces una tensión que obstruye la consecución de ellos. Esta situación es común para América, así lo señala la Organización Panamericana de la Salud (OPS) en el 2010 al decir que los sistemas de salud en la región, se caracterizan por altos niveles de fragmentación de los servicios. La experiencia acumulada demuestra que esto genera dificultades en el acceso a los servicios, en la prestación de baja calidad técnica y en el uso irracional e ineficiente de los recursos disponibles, de forma que provoca un incremento innecesario de los costos de producción y una baja satisfacción de los ciudadanos con los servicios recibidos.
Esta excesiva división de las organizaciones de salud se manifiesta de múltiples formas en los distintos niveles del sistema, comenzando por su desempeño general donde la fragmentación se manifiesta en una falta de coordinación entre los distintos niveles y espacios de atención lo que provoca una duplicación de servicios y de infraestructura, haciendo a la capacidad instalada ociosa, al no utilizar los espacios de forma eficaz especialmente en los hospitales. Por otra parte, en la experiencia de las personas que utilizan el sistema la fragmentación se expresa como falta de acceso a los servicios con una pérdida de continuidad de la atención lo que supone una incongruencia de los servicios con las necesidades de los usuarios (OPS, 2010). Esta situación había sido descrita en el 2008 por la misma institución cuando confirmó que había una necesidad urgente de abordar el problema de la fragmentación, promoviendo la iniciativa de generar Redes Integradas de Servicios de Salud (RISS), que potencien a las organizaciones de salud con mecanismos de coordinación asistencial, estandarización de procesos y sistemas de información integrados, entre otros.

Esta evaluación hecha desde el organismo cuya misión es velar por la salud de la población de la región tiene la validez del saber experto y la indicación de la inminente necesidad de innovar en los servicios de salud a nivel organizacional se sustenta desde el saber propio de la cultura organizacional, así Henry Mintzberg (1979), estratega empresarial, plantea que toda actividad humana organizada, desde la formación de las piezas de barro hasta el envío del hombre a la Luna, requiere dos requisitos a la vez fundamentales y opuestos: la división del trabajo y la coordinación de los mismos. Donde la estructura de la organización se define simplemente como el conjunto de todas las formas en que se divide el 
trabajo, distintas tareas que posteriormente deben ser coordinadas. Para lograr esta coordinación del trabajo, una organización debe utilizar cinco mecanismos fundamentales: adaptación mutua, supervisión directa, normalización de los procesos de trabajo, normalización de los resultados y normalización de las habilidades.

Para este ensayo que reflexiona en la gestión del cuidado en un sistema de salud que exige innovación organizacional se articula el saber propio de la enfermería con el saber de organizacional, de modo que de los cinco mecanismos, es la adaptación mutua la que cobra un interés especial por la coherencia que tiene con la lógica del cuidado descrita en la disciplina de Enfermería, un cuidado situado en un contexto de relaciones interpersonales donde se alcanza la coordinación del trabajo mediante la simple comunicación informal, así los "operadores" como los denomina la teoría organizacional o miembros de un equipo como se llaman en salud, rápidamente coordinan sus acciones unos con otros. Mintzberg señala que la adaptación mutua se presenta en pequeñas organizaciones, pero cuando se presenta en grandes organizaciones se requiere de un directivo integrador como los jefes de unidades en hospitales encargados de integrar las actividades de médicos, de enfermeras y del staff de apoyo.

Siendo el objetivo de la asistencia sanitaria proveer a los pacientes, en el nivel asistencial más adecuado y de la forma más eficiente posible de aquellos servicios que mejor preserven o restauren su estado de salud. Sin embargo, la práctica clínica implica para el proveedor la toma diaria de decisiones y la realización de prácticas que, por diferentes razones, están sujetas a variabilidad (Lorenzo S., Mira J., Sánchez E., 2000) debido a que el nivel de incertidumbre es amplio por ser cada caso único, si bien existe un nivel de precisión que permite predecir, normar y planificar la práctica clínica, la complejidad de las circunstancias que rodean el caso favorecen la variabilidad y exigen desarrollar acciones adecuadas a una dinámica específica, el negar esta versatilidad en la práctica clínica puede generar actividades inadecuadas o errores que incluso pueden ocasionar daño al paciente. Es por esto que para Enfermería la gestión del cuidado, como señala Suzanne Kérouac (1996), es un "proceso creativo, dirigido a movilizar los recursos humanos y los del entorno con la intención de mantener y favorecer el cuidado de la persona que, en interacción con su entorno, vive experiencias de salud" precisando una coordinación dinámica y flexible que permita satisfacer las necesidades de un cuidado complejo.

Zárate (2005) refiere que otro aspecto de la Gestión del cuidado inherente a la práctica de enfermería y que merece ser señalado, se trata de la colaboración intradisciplinaria que comprende la consulta entre colegas enfermeras, igual que la participación de enfermeras clínicas en el desarrollo de conocimientos y en la formación de futuras enfermeras. La colaboración intradisciplinaria puede tener un impacto importante en la continuidad y la calidad de los cuidados $y$ es algo que las enfermeras poco realizamos. La identificación de enfermeras expertas y las consultas entre colegas enfermeras, los conocimientos compartidos, refuerzan el potencial y la competencia del grupo profesional, mientras que los conocimientos celosamente guardados en un escritorio o en la mente de alguna enfermera experta no contribuyen a una práctica de calidad y colaboración. El punto de inicio de esta colaboración es reconocernos nosotras mismas como expertas y enriquecer de manera constante nuestros conocimientos y habilidades.

De acuerdo con lo señalado en la Norma Administrativa $\mathrm{N}^{\circ} 19$, "Gestión del cuidado de enfermería para la atención cerrada", del Ministerio de Salud de Chile: la "Gestión del cuidado de enfermería", entendida como el ejercicio profesional de la enfermera sustentada en su disciplina, "la ciencia del cuidar", se define como la aplicación de un juicio profesional en la planificación, organización, motivación y control de la provisión de cuidados, oportunos, seguros, 
integrales, que aseguren la continuidad de la atención y se sustenten en las políticas y lineamientos estratégicos de la institución. La Gestión del cuidado de enfermería es, entonces, uno de los componentes básicos que contribuyen al desarrollo de la Gestión Clínica Hospitalaria, concebida como el uso más adecuado de los recursos profesionales, humanos, tecnológicos y organizativos para otorgar el mejor cuidado a las personas. Por lo tanto, su fin último es ofrecer a los usuarios los mejores resultados posibles en la práctica diaria, acorde con la información científica disponible que haya demostrado su capacidad para cambiar de manera favorable el curso clínico de la enfermedad y que considere la mejor administración de los recursos, los menores inconvenientes y costos para el usuario y para la sociedad en su conjunto.

Se señala además, que en consecuencia, la Gestión Clínica se sustenta en dos pilares que requieren aplicar conocimientos tanto del ámbito clínico como gerencial, a saber: la gestión médica de "curar" y la gestión de enfermería de "cuidar", dichos ejes o pilares de la atención sanitaria son además enriquecidos con la participación de otras disciplinas que ofrecen respuestas a los problemas de salud de la población, dándoles el carácter multidisciplinario al proceso de atención de salud de acuerdo a las necesidades del individuo, familia y/o comunidad.

Es así como los cuidados de enfermería evolucionan hacia un hacer más definido, estructurado y evaluado según los principios de la gestión científica. El plan de cuidados es la herramienta de trabajo de la enfermera para la planificación y continuidad de los cuidados y, por lo tanto, para la gestión de los mismos. Independientemente de las teorías en las que se fundamente, este plan asegura que cada paciente recibe los mejores cuidados, con la mejor evidencia, pero también individualizados a lo que él necesita (Rich M., Cubillo, G., Barberá M., Bravo, M.C., 2003).
Nos encontramos, entonces, frente a un gran reto en nuestro quehacer, gestionar el cuidado de enfermería rompiendo las barreras que generan fragmentación y lograr la coordinación del trabajo, pero ¿Cómo podemos lograr esto, en la práctica en nuestras tareas diarias?

Troncoso MP. \& Suazo SV. (2007) han demostrado que los pacientes valoran más los aspectos del cuidado que tienen relación con la comunicación, el afecto y la atención que se le presta dentro de las instituciones hospitalarias, es decir, un cuidado humanizado y transpersonal. Pero esta comunicación no solo es entre enfermera "paciente" o enfermera- "cliente", sino que es fundamental en los equipos de trabajo para mantener la continuidad de la información y, por ende, la continuidad del cuidado siendo un elemento indispensable en la calidad de las relaciones, en la cual ocurre un intercambio de mensajes, enviados, recibidos, compartidos y retroalimentados.

Por otra parte, Ulla S., Arranz P., Argüello MT. \& Cuevas C. (2002) determinaron las dificultades de comunicación percibidas por profesionales de enfermería en un hospital general, concluyendo que la dificultad para la comunicación con los médicos es mucho mayor que la observada para la comunicación con los profesionales en enfermería, siendo el grado de fluidez de la comunicación un aspecto fundamental en la coordinación dentro de los equipos que puede derivar en situaciones de malestar o insatisfacción entre los miembros del equipo, generando un factor de riesgo para posteriores cuadros de estrés asistencial, mayor ausentismo laboral y mayor índice de rotación.

Es por esto que la comunicación se transforma en un eje central de la coordinación, para Jody Hoffer Gittel (2009) la comunicación se enmarca en una coordinación relacional, entendiendo esta como el proceso que comprende la interacción entre la comunicación y las relaciones llevadas a cabo con el propósito de la integración de tareas. Es una teoría emergente para la comprensión de la 
dinámica relacional de coordinar el trabajo. Es interesante detenerse en esta investigación que inicialmente estudia el desempeño de la coordinación de las relaciones en The Southwest Airlines y el impacto de esta en otro tipo de industria como es mercado de la salud. En este caso, comprobamos que la atención sanitaria se ve potenciada con la participación de otras disciplinas como la economía política y las ciencias políticas en las áreas de la gestión.

En la teoría de la coordinación relacional hay tres dimensiones de las relaciones que son parte integral del proceso de coordinación: conocimientos compartidos, objetivos compartidos y respeto mutuo.

Respecto a la comunicación hay cuatro dimensiones: comunicación frecuente, comunicación oportuna, comunicación precisa y solución de problemas de comunicación. Esta teorí a ha sido desarrollada y probada en el contexto de los viajes aéreos, la atención quirúrgica y cuidados de larga duración, y se espera generalizar la teoría coordinación relacional a procesos en los que varios proveedores se dedican a llevar a cabo tareas altamente interdependientes en condiciones de incertidumbre y limitaciones de tiempo de trabajo.

La figura 1 ilustra los múltiples proveedores que participan en un proceso de atención de pacientes. En los contextos donde ha sido explorada, la coordinación relacional parece tener un impacto significativo y positivo en las medidas claves de rendimiento, incluyendo tanto la calidad y la eficiencia lo que permite que la gente responda a información nueva de una forma espontánea y coordinada. No obstante, la frecuencia de la comunicación en la atención de pacientes no garantiza la calidad de esta, esto supone que la atención al paciente estará mal coordinada, cuando la comunicación, -no importa con qué frecuencia- se retrasa, es inexacta o se centra en focos diferentes.
Figura 1: Atención a los pacientes: un reto de coordinación

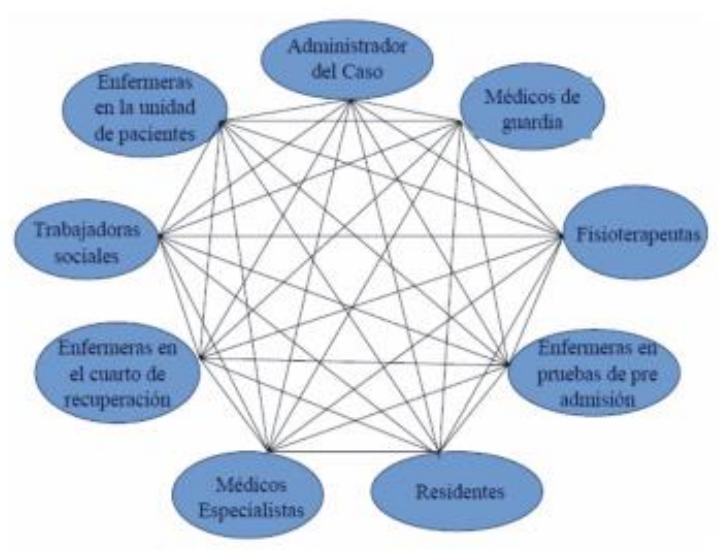

Fuente: Gittell, Jody Hoffer, 2009

El siguiente cuadro muestra las distintas dimensiones de la teoría y el fundamento de cada una de ellas.

Figura 2: Dimensiones de la coordinación relacional

\begin{tabular}{l|l|}
\multicolumn{1}{c|}{ Dimensión } & \multicolumn{1}{c|}{ Fundamento } \\
$\begin{array}{l}\text { Comunicación } \\
\text { frecuente }\end{array}$ & $\begin{array}{l}\text { Ayuda a construir relaciones a través de la familiaridad que } \\
\text { se da por la comunicación repetida }\end{array}$ \\
\hline $\begin{array}{l}\text { Comunicación } \\
\text { oportuna }\end{array}$ & $\begin{array}{l}\text { La comunicación tiene que ser en el momento adecuado } \\
\text { para que sea de calidad. }\end{array}$ \\
\hline $\begin{array}{l}\text { Comunicación precisa } \\
\text { La información entregada debe ser precisa. La información } \\
\text { inexacta puede llevara errores, retrasos y disminución de la } \\
\text { eficacia. }\end{array}$ \\
\hline $\begin{array}{l}\text { Soluciónde problemas } \\
\text { de comunicación }\end{array}$ & $\begin{array}{l}\text { Una coordinación eficaz requiere que los participantes se } \\
\text { incorporen a la resolución de problemas de comunicación. }\end{array}$ \\
\hline $\begin{array}{l}\text { Relaciones de respeto } \\
\text { mutuo }\end{array}$ & $\begin{array}{l}\text { En todo el proceso de coordinación debe existir respeto entre } \\
\text { los participantes. La falta de respeto es posible fuente de } \\
\text { división. }\end{array}$ \\
\hline $\begin{array}{l}\text { Conocimiento } \\
\text { compartido }\end{array}$ & $\begin{array}{l}\text { Debe existir un alto grado de conocimientos compartidos en } \\
\text { relación con las demás tareas. }\end{array}$ \\
\hline Objetivos compartidos & $\begin{array}{l}\text { Para que exista coordinación efectiva debe existir un nivel } \\
\text { elevado de objetivos comunes entre los participantes. }\end{array}$ \\
\hline Fuente: Gittell, Jody Hoffer, 2009 \\
\hline
\end{tabular}

Es así como lo señalado por Gittell J.H. (2009) la coordinación relacional permite a los empleados organizar más eficazmente su trabajo con los demás, para lograr resultados de calidad superior utilizando recursos de manera más eficiente; por ejemplo, en un hospital, lograr mayor calidad de la atención percibida por el paciente, junto con estadías más cortas. En una economía cada vez más competitiva, las empresas de salud, tienen que afrontar mayores dificultades y exigencias de distintas índoles entre las cuales destacan los aspectos éticos de su servicio, esto implica para este tipo de organizaciones un reto superior 
respecto a otras industrias. Hasta el momento, se han documentado los efectos de rendimiento de coordinación relacional de la industria aérea, en la industria hospitalaria y en la industria de cuidados ambulatorios. Pero el logro de coordinación relacional depende de la adopción de un conjunto de prácticas de organización que apoyan su desarrollo, la ciencia de enfermería en su marco epistemológico contiene aspectos claves que pueden favorecer dichas prácticas, debido a que considera como la base del cuidado una relación interpersonal, que ocupan espacios diferentes en la interacción, la relación profesional de la salud con la persona que necesita cuidado, es el encuentro de dos personas, dos realidades en diferentes situaciones y con diferentes saberes en el contexto del respeto y la confianza.

Cuando los médicos, los profesionales de enfermería, las terapeutas, los técnico, otros trabajadores del equipo de salud y administrativos están unidos por objetivos comunes con intercambio de conocimientos y respeto mutuo, la comunicación tiende a ser más frecuentes, oportuna, precisa debido a que se centra en la solución de problemas, lo que les permite ofrecer rentabilidad y una atención de alta calidad a los pacientes. Sin embargo, se puede observar que en ocasiones entre estos diversos proveedores faltan objetivos comunes, no hay intercambio de conocimientos y se altera el respeto mutuo, incluso trabajando con los mismos pacientes, su comunicación es poco frecuente, inoportuna, inexacta, y están más centrados en culpar por los errores que en la resolución de problemas. Cuando esto sucede, se pierden esfuerzos, recursos y no se ofrece una atención de alta calidad. Las relaciones son un ingrediente esencial de cualquier solución viable al problema de coordinación, especialmente en gestionar el cuidado de los pacientes (Gittell J.H.,2009).

La autora señala que los hospitales, al igual que otras empresas, también están bajo la presión de reducir costos de personal, pero esta reducción la hacen a través de un mix de personal más barato con una combinación de capacidades menores, de forma que la asignación de algunas tareas no es acorde a las capacidades de este personal, es en este caso, donde la coordinación debe ser aún mayor aumentando el número de transferencias en el equipo de salud.

Los hospitales no solo tienen las presiones para incrementar la calidad y reducir los costos, sino que además, por generar un modelo de gestión del cuidado que debe responder a estándares nacionales e internacionales de calidad y seguridad (Gittell J.H., 2009).

\section{Conclusiones}

La adaptación mutua, entendida como la coordinación del trabajo mediante mecanismos de coordinación que se potencian con la comunicación entre profesionales que participan en un mismo proceso asistencial, es más eficaz para coordinar la atención de problemas de salud complejos que presentan un elevado nivel de incertidumbres e interdependencias.

El aporte que nos hace Jody Hoffer Grittel, desde el área de la industria y los negocios para optimizar el quehacer del equipo de salud, es relevante y convergente con el significado de la gestión del cuidado para enfermería, nos entrega elementos claves que potencian el saber de la disciplina de enfermería para brindar un cuidado de calidad que satisfaga las expectativas del usuario y que además optimice recursos logrando favorecer el costo- efectividad para las instituciones.

\section{Bibliografía}

Colegio de Enfermeras (2007) Gestión del cuidado en Atención Primaria

www.colegiodeenfermeras.cl

Recuperado 22 de febrero 2011 http:/ / www.colegiodeenfermeras.cl/info.asp?Ob= $1 \& I d=830$ 
Gittell, J. H. (2009) High performance healthcare: using the power of relationships to achieve quality, efficiency and resilience. New York: McGraw-Hill.

Lorenzo S., Mira J.J., Sánchez E. (2000) Gestión de calidad total y medicina basada en la evidencia. Medicina Clínica, 1 14, 12,460-463.

Ministerio de Salud de Chile, Subsecretaría de Redes Asistenciales. Norma General Administrativa №19, "Gestión del Cuidado de Enfermería para La Atención Cerrada. 14 de Diciembre de 2007.

Santiago de Chile. Recuperado 22 de febrero 2011 www.juridico 1.minsal.cl/RESOLUCION_1127_07.

doc

Mintzberg H. (1991). Mintzberg y la organización. Madrid: Díaz de Santos

Mintzberg H. (1999). La estructuración de las organizaciones. Barcelona: Ariel.

Olmedo M., De Blas S., Mestre RP., Puente, M.D.
Rodríguez P. (2010). Sistemática para la protocolización de los cuidados de enfermería. Revista Calidad asistencial, 25, 268-274.

Poblete M. \& Valenzuela S. (2007). Cuidado humanizado: un desafío para las enfermeras en los servicios hospitalarios. Acta Paulista de Enfermagem [online]. 2007, vol.20, n.4, pp. 499503.ISSN 0103-2100. Recuperado 22 de febrero 2011 http: / / www.scielo.cl/scielo.php?pid=S07179 $5532010000100004 \&$ script $=$ sci_arttext

Rich M., Cubillo G., Barberá M., Bravo, M.C. (2003). Estimación de los costes de enfermería en el Proceso de la enfermedad pulmonar obstructiva Crónica (EPOC). Enfermería Global, 2, 1-6.

Zarate R. (2004). La Gestión del Cuidado de Enfermería. Index Enferm [revista en la Internet]. 2004 Jun [citado 2011 Ene 20]; 13(44-45): 42-46. Disponible en: doi: $10.4321 / 51132$ 12962004000100009

http:/ / scielo.isciii.es/scielo.php?script=sci_arttex t\&pid=S1 $13212962004000100009 \& \operatorname{lng}=e s$ 